\title{
Le Mystère de saint Clément de Metz, Édition critique par Frédéric Duval
}

\section{Maria Colombo Timelli}

\section{(2) OpenEdition}

1 Journals

\section{Édition électronique}

URL : https://journals.openedition.org/studifrancesi/3034

DOI : 10.4000/studifrancesi.3034

ISSN : 2421-5856

\section{Éditeur}

Rosenberg \& Sellier

\section{Édition imprimée}

Date de publication : 1 juillet 2013

Pagination : 438-439

ISSN : 0039-2944

\section{Référence électronique}

Maria Colombo Timelli, «Le Mystère de saint Clément de Metz, Édition critique par Frédéric Duval », Studi Francesi [En ligne], 170 (LVII | II) | 2013, mis en ligne le 30 novembre 2015, consulté le 02 février 2023. URL : http://journals.openedition.org/studifrancesi/3034 ; DOI : https://doi.org/10.4000/ studifrancesi.3034

Ce document a été généré automatiquement le 2 février 2023.

\section{(c) () $\odot$}

Creative Commons - Attribution - Pas d'Utilisation Commerciale - Pas de Modification 4.0 International - CC BY-NC-ND 4.0

https://creativecommons.org/licenses/by-nc-nd/4.0/ 


\title{
Le Mystère de saint Clément de Metz, Édition critique par Frédéric Duval
}

\author{
Maria Colombo Timelli
}

\section{RÉFÉRENCE}

Le Mystère de saint Clément de Metz, Édition critique par Frédéric DUVAL, Genève, Droz, 2011, («Textes Littéraires Français», 608), pp. 815.

1 Cette édition du Mystère de saint Clément répond à deux exigences, l'une plus traditionnelle - mettre à la disposition des lecteurs un texte dramatique quasiment inconnu -, l'autre méthodologique, et c'est là sans doute que repose son intérêt principal. La situation textuelle est en effet la suivante: une édition confidentielle fournie par Charles Abel en 1861 (tirée à 141 exemplaires, maintenant numérisée), qui ne répond évidemment pas aux critères scientifiques d'aujourd'hui, et quelques feuillets de ses épreuves; une thèse allemande de 1909 (de Fritz Tinius, sous la direction d'Edmund Stengel), qui fournit des matériaux philologiques importants, à savoir l'édition d'un millier de vers (le Mystère en compte un peu plus de 9000), mais aussi un relevé des divergences entre l'édition Abel et le manuscrit; un manuscrit unique conservé à Metz, qui constituait sans doute un «manuscrit de théatre original» (p. 23) au sens qu'attribue à cette formule D. Smith, tout en s'agissant de la copie d'un texte antérieur, disparu dans l'incendie de 1944; quatre lignes de ce même manuscrit reproduites en fac-similé par C. Abel. C'est dire que F. Duval ne disposait pas d'un texte de base fiable: voilà pourquoi son travail constitue une véritable "gageure philologique», selon ses propres mots (p. 12).

2 L'Introduction est substantielle. Il s'agissait en premier lieu de faire le point sur les «témoins» du texte, aussi divers soient-ils, comme on vient de le rappeler (pp. 19-28). L'analyse (pp. 28-34) permet d'avoir une idée claire de l'intrigue. Des pages essentielles sont aussi consacrées à la légende de saint Clément, premier évêque de Metz, dont on conserve une série de Vitae en latin et six Vies en français échelonnées du $\mathrm{XIV}^{\mathrm{e}} \mathrm{au} \mathrm{XVI}^{\mathrm{e}}$ 
siècle, celles-ci dérivées de la Vita quarta; tout cela prouve, si besoin était, la vivacité du culte de ce saint entre la fin du Moyen Âge et le siècle de la Renaissance. La connaissance de ce cadre permet à $\mathrm{F}$. Duval d'affirmer avec une relative sûreté que le fatiste s'est probablement inspiré de la tradition française de la Vita quarta (p. 43). L'analyse littéraire et dramaturgique (pp. 46-83) approfondit l'examen de ce texte «à forte potentialité scénique» (p. 48); en effet, l'auteur du Mystère a scrupuleusement respecté la légende hagiographique en y ajoutant des scènes bien identifiables, attachées à la tradition théâtrale et à ses conventions: $\mathrm{F}$. Duval rappelle en particulier le traitement du temps et de l'espace, l'introduction de scènes de genre, profanes ou religieuses, célestes ou infernales, qui rendent vivace la représentation (on rappellera quelques guérisons miraculeuses, des sermons, les rencontres entre des messagers et des vilains, des scènes à la taverne); l'auteur anonyme s'avère aussi capable d'approfondir la psychologie des personnages secondaires. La pièce est structurée en tableaux, les mansions permettant de représenter les nombreux déplacements, grâce aussi aux personnages de transition, car le messager joue dans ce cas un rôle de premier plan. Parfaitement conscient du caractère purement conjectural (p. 65) de son discours sur la mise en scène, $F$. Duval peut néanmoins souligner les nombreuses indications scéniques présentes tant dans les didascalies que dans les dialogues. L'ensemble comprend environ 9400 octosyllabes à rimes plates, ce qui devait correspondre à une représentation durant une journée et demie (p. 70); quelque 120 personnages entraient en scène, pour un nombre évidemment inférieur d'acteurs; peu de décors complexes, peu de mansions (p. 74), quelques objets emblématiques (p. 76), des costumes (pp. 76-78), constituent l'arsenal d'une pièce qui avait pour but de réunir le public messin autour du saint patron de la ville, de l'instruire, de l'édifier, mais également de lui procurer un divertissement au sens étymologique du mot (p. 82).

3 L'analyse linguistique tient évidemment compte de la situation textuelle, et ne s'arrête que sur les phénomènes avérés et récurrents appartenant à la scripta messine du $\mathrm{xv}^{\mathrm{e}}$ siècle (pp. 83-104). Le paragraphe consacré à la versification - déjà étudiée par Henri Chatelain dans ses Recherches de 1907, puis par F. Tinius - rappelle rapidement les problèmes essentiels liés aux rimes et au rythme (pp. 105-112).

4 L'auteur du Mystère, certainement lorrain sinon messin, demeure nécessairement anonyme (p. 112), alors que la datation du texte peut être fixée approximativement, et avec une grande prudence, peu après 1439 , bien que le manuscrit soit plus tardif (entre 1467 et 1477) (pp. 113-119).

5 Le but de cette édition est bien entendu de donner à lire un texte qui, sans être un chef d'œuvre, mérite bien d'être redécouvert. Un des mérites de F. Duval est de rendre possible à tout moment le retour à l'édition Abel (ses italiques sont conservés, les leçons éventuellement rejetées ou corrigées sont indiquées dans l'apparat en bas de page) et le renvoi aux leçons corrigées par Tinius (intégrées dans le texte et également mentionnées en bas de page). La stratégie d'édition et de correction adoptée par M. Duval est très clairement exposée, et se fonde sur une étude extrêmement approfondie du travail de son prédécesseur (pp. 119-157): elle repose sur des conjectures, certes, mais suit des principes philologiques rigoureux. Les critères pour la présentation du texte sont aussi exposés très clairement (pp. 157-160).

6 Le texte du Mystère est établi avec un grand soin et se lit très agréablement aux pp. 163-624; outre l'apparat critique déjà mentionné, il est accompagné d'un nombre important de notes (pp. 625-720) ponctuellement signalées dans les vers selon un 
double système de repérage qui sépare les notes concernant spécifiquement l'établissement du texte et celles qui se rapportent à d'autres aspects, linguistiques entre autres. Suivent l'index des personnages (par ordre d'apparition: pp. 721-725), un index des noms (par ordre alphabétique: pp. 727-737), le glossaire (en même temps riche et essentiel, à compléter par un usage intelligent du DMF: pp. 739-813). On regrette un peu l'absence d'une bibliographie: les renvois fournis aux pp. 24-25 se limitent aux titres essentiels, alors que la bibliographie citée dans les notes est bien plus abondante.

7 Quelques notes de lecture sur trois fragments (vv. 1-1045, 4045-4795, 8284-fin). Pour ce qui est de la transcription, je n'ai que quelques petites observations: ponctuation sans doute à revoir aux vv. 548-549; alternance de l'initiale minuscule (vv. 4047 et 4247) et majuscule (préférable: vv. 735, 4323) pour Fils, deuxième personne de la Trinité. Plus généralement, j'aurais adopté la graphie s'y vous plaist / s'y vous agree plutôt que la graphie agglutinée sy (vv. 4616, 8415, 8454, 8779, 8888, 8928 9039; d'ailleurs, s'y se lit au v. 4488); de même, j'aurais introduit une apostrophe aux vv. 4716 (s'y ne tient au masson) et 8358 (ne t'y fains mie). Toujours pour les diacritiques, aït au v. 818 (Dieu vous ait! Dieu vous ait! Proudomme) rend le vers hypermétrique, alors que la graphie sans tréma est bien adoptée au v. 4680 (même formule de souhait: Dieu vous aist, Dieu vous aist, prodome). Remarquons enfin que saint Ongnon (v. 50) est ignoré du Dictionnaire érotique de Rose Bidler (Montréal, Ceres, 2002: s.v. saint).

Cette édition fournit un excellent exemple méthodologique que l'on pourra certainement appliquer à d'autres textes: le cas du Mystère de saint Clément (manuscrit unique perdu, éditions pré-scientifiques peu fiables, témoignages indirects) est loin d'être isolé. Je me permets toutefois une remarque: l'exemple de Gliglois (cité à l'appui p. 12, note 14) n'est pas adéquat, puisque le manuscrit de Turin, BNU L.IV.33 - quoi qu'en disent les deux éditeurs récents de ce roman - n'a pas disparu dans l'incendie qui a ravagé cette bibliothèque en 1904 (cf. «Studi Francesi», 151, 2007, pp. 147-149). 